\title{
Distribution of Two Distinct Genotypes of Citrus Greening Organism in the Ryukyu Islands of Japan
}

\author{
Kenta TOMIMURA ${ }^{1}$, Noriko FURUYA ${ }^{2}$, Shin-ichi MIYATA ${ }^{2}$, \\ Akiko HAMASHIMA ${ }^{3}$, Hiroaki TORIGOE ${ }^{3}$, Yuko MURAYAMA ${ }^{4}$, \\ Shinji KAWANO ${ }^{4}$, Mitsuru OKUDA ${ }^{5}$, Siti SUBANDIYAH ${ }^{6}$, \\ Hong-Ji SU ${ }^{7}$ and Toru IWANAMI ${ }^{2 *}$ \\ ${ }^{1}$ Kuchinotsu Citrus Research Station, National Institute of Fruit Tree Science, National Agriculture and \\ Food Research Organization (NARO) (Minami-shimabara, Nagasaki 859-2501, Japan) \\ ${ }^{2}$ National Institute of Fruit Tree Science, NARO (Tsukuba, Ibaraki 305-8605, Japan) \\ ${ }^{3}$ Fruit Tree Department, Kagoshima Prefectural Institute for Agricultural Development \\ (Tarumizu, Kagoshima 891-2112, Japan) \\ ${ }^{4}$ Okinawa Prefectural Agricultural Research Center (Itoman, Okinawa 901-0336, Japan) \\ ${ }^{5}$ National Agricultural Research Center for Kyushu Okinawa Region, NARO \\ (Koshi, Kumamoto 861-1192, Japan) \\ ${ }^{6}$ Department of Entomology and Plant Pathology, Gadjah Mada University (Yogyakarta, 55281, Indonesia) \\ ${ }^{7}$ Department of Plant Pathology and Entomology, National Taiwan University (Taipei, 10764, Taiwan)
}

\begin{abstract}
The Asian type "Candidatus Liberibacter asiaticus" (Las, citrus greening organism) is severely damaging citrus production in Asia including Japan. Our previous study suggested that the bacteriophagetype DNA polymerase region (DNA pol) would be useful for molecular differentiation in different Southeast Asian Las isolates. Moreover, Las isolates originated from most of the Southeast Asian regions harbor the DNA pol gene, whereas Japanese ones lack this region. These preliminary findings lead us to a hypothesis that all Japanese isolates lack DNA pol. To try this hypothesis, we collected citrus leaf samples infected with Las throughout the Ryukyu Islands, and examined them by a duplex PCR that could simultaneously amplify two DNA fragments of DNA pol and nusG-rplK operon of Las. The duplex PCR was applied to the collection of 65 Las isolates. Both DNA pol and nus G-rplK operon were successfully amplified from nine isolates, whereas only nus G-rplK operon was amplified with the other 56 isolates. These nine isolates with DNA pol originated from the Hateruma, Irabu, Kohama, Miyako, Tarama, and Yonaguni Islands, which are geographically close to Taiwan. The nucleotide sequence of DNA pol of these nine isolates was identical, and was also the same as four Taiwanese isolates reported previously. These results suggest that Japanese Las isolates comprise at least two distinct genotypes, and the genotype that had DNA pol is highly homogeneous.
\end{abstract}

Discipline: Plant disease (Plant pathology)

Additional key words: citrus greening disease, Diaphorina citri, duplex PCR, Huanglongbing

\section{Introduction}

Citrus greening (Huanglongbing) is one of the most devastating citrus diseases in many parts of the world (Asia, North America, South America, and Africa). Citrus greening is a threat to the citrus industry ${ }^{4}$. The causal agents of this disease (citrus greening organism) are Candidatus Liberibacter spp., which are gram negative, phloem-limited bacteria that cannot be cultured. The pathogens are mainly transmitted by the psyllids Trioza erytreae (T. erytreae) in Africa ${ }^{1}$ and Diaphorina citri ( $D$. citri) in Asia, North America and South America ${ }^{5}$. Compared with $D$. citri, T. erytreae is found in cooler areas and at higher altitudes. D. citri is more widely spread in warmer lowlands in tropical and subtropical areas. Contaminated plant materials used for propagation of nursery plants also transmit these pathogens. Three Ca. Liberibacter spp. that act as pathogens have been identified: $\mathrm{Ca}$. Liberibacter asiaticus (Las), $\mathrm{Ca}$. Liberibacter africanus ${ }^{9}$ and $\mathrm{Ca}$. Liberibacter americanus ${ }^{3}$.

Infected citrus trees show various symptoms such

*Corresponding author: e-mail tiwsw37@affrc.go.jp

Received 2 March 2009; accepted 2 June 2009. 
as yellowing and blotchy mottling on their leaves and fruit. Infected trees and branches suffer heavy leaf drop followed by out-of-season flushing and blossoming, and in severe cases, dieback. However, none of the symptoms are specific to citrus greening ${ }^{2}$. In Japan, this disease was found for the first time on Iriomote Island in $1988^{12}$. Subsequently, it was found on Okinawa Main Island in $1994^{10}$, Yoron Island in $2002^{7}$, and Okinoerabu, Tokunoshima and Kikai Islands in $2003^{15}$. The incidence survey in Okinawa Prefecture revealed that Las was found throughout the Okinawa Islands excluding North and South Daito Islands ${ }^{13}$. The transmission vector, D. citri was apparently distributed throughout the Ryukyu Islands, and Las positive psyllids were found in most of these islands ${ }^{14}$. These observations indicate that the disease is spreading northward gradually in these areas. North of these islands, there is a main island, Kyushu, which has the main citrus production areas of this region, and northbound dispersion of the disease poses a great threat to citrus cultivation there.

Assessment of genetic diversity provides a framework for understanding the taxonomy, population structure and dynamics of phytobacteria. It also provides a key for devising sensitive, specific and rapid methods for detecting the pathogen, diagnosing plant disease, and managing disease risk ${ }^{11}$. Methods to distinguish Las isolates are fundamental for ecologic and epidemiologic studies. We reported genetic diversity of Las in Southeast Asia previously $^{16}$. The degree of genetic diversity was different in each gene region of Las, and the most diverse gene region was the bacteriophage-type DNA polymerase region (DNA pol). The Ryukyu Islands of Japan are located near Taiwan, and the closest island, Yonaguni, is approximately $100 \mathrm{~km}$ apart from Taiwan. This close geographical situation has made us postulate that genotypes of Las in the Ryukyu Islands are similar to those of Taiwan.

In this paper, we determined the Las genotypic distribution in the Ryukyu Islands of Japan using the duplex PCR developed in this study. Contrary to the initial assumption, the results showed that there are two distinct genotypes in the population structure of Las in the Ryukyu Islands of Japan. One genotype occurs also in Taiwan and another genotype is unique to Japan. The results also indicated that one of the two genotypes may constitute an absolutely homogeneous population related to Taiwanese isolates. We also discuss the relationship between the genotypic composition and geographical origin.

\section{Materials and methods}

\section{Sample collection and preparation}

Leaf samples were collected from infected citrus trees in different orchards throughout the Ryukyu Islands of Japan (Table 1). Total DNA was extracted from about 0.2 $\mathrm{g}$ of the leaf midrib from the infected citrus tree using a DNeasy Plant Mini kit (Qiagen, Valencia, USA) according to the manufacturer's instructions. The extracted DNA was suspended in 20-200 $\mu$ l of TE buffer (10 mM Tris$\mathrm{HCl}, 1$ mM EDTA, pH 8.0).

\section{Duplex polymerase chain reaction}

Genotypes of Las isolates were determined by the duplex PCR based on DNA pol and nusG-rplK operon. DNA pol was chosen as a variable region to differentiate isolates, while nus G-rplK operon was selected as a conserved region among isolates to verify the presence of Las. One primer set of DNA pol was previously reported as GODNPFW1 (5'-TCCTGAGAATTACACACAAAC-3') and GODNPRV1 (5'-TCTAAGTCTATCCTGTAACCC$\left.3^{\prime}\right)^{16}$. The other primer set of nusG-rplK operon was previously reported as MHO353 (5'-GTGTCTCTGAT GGTCCGTTTGCTTCTTTTA-3') and MHO354 (5'GAACCTTCCACCATACGCATAGCCCCTTCA-3') $)^{8}$. Genotyping by the duplex PCR using both of these primer sets was assessed by the following amplicons: 988 bp and 627 bp for DNA pol and nusG-rplK operon, respectively. The PCR reactions were performed using an iCycler thermal cycler (Bio-Rad Laboratories Hercules, CA, USA) in $20 \mu 1$ reaction mixture volumes containing $2 \mu \mathrm{l}$ of DNA template, $1 \mu \mathrm{M}$ of each primer, $200 \mu \mathrm{M}$ of dNTP mixture, $1 \times$ PCR buffer, and 0.5 units of Ex Taq DNA polymerase Hot Start Version (TaKaRa, Japan). The thermal cycling conditions were as follows: initial denaturing at $94^{\circ} \mathrm{C}$ for $2 \mathrm{~min}$; 40 cycles of denaturing at $94^{\circ} \mathrm{C}$ for $30 \mathrm{~s}$, annealing at $50^{\circ} \mathrm{C}$ for $30 \mathrm{~s}$, extension at $72^{\circ} \mathrm{C}$ for $1 \mathrm{~min}$; and a final extension step at $72^{\circ} \mathrm{C}$ for $5 \mathrm{~min}$. The amplified PCR products were separated by electrophoresis in $1.5 \%$ agarose gels. Gels were stained with $0.5 \mu \mathrm{g} / \mathrm{ml}$ ethidium bromide, and visualized under a UV transilluminator.

\section{Sequence analysis of bacteriophage-type DNA polymerase region}

Among the analyzed isolates in this study, nine isolates that possessed the DNA pol were selected, and the sequence of the DNA pol and flanking regions were determined as described previously ${ }^{16}$ and compared among isolates. Sequence data were assembled using BioEdit version $5.0 .9^{6}$.

\section{Results}

Duplex PCR results would show that the isolates with DNA pol produce two DNA bands of DNA pol and nusG$r p l \mathrm{~K}$ operon after agarose gel electrophoresis, whereas the 
Table 1. Isolates of Candidatus Liberibacter asiaticus used in this study

\begin{tabular}{|c|c|c|c|c|}
\hline & Isolate & Code & Location & Year of collection \\
\hline \multirow[t]{7}{*}{ 1) Kikai Island } & Kikai-130 & $\mathrm{Hm} 1$ & Oasato, Kikai, Kagoshima & 2006 \\
\hline & Kikai-145 & $\mathrm{Hm} 2$ & Oasato, Kikai, Kagoshima & 2006 \\
\hline & Kikai-147 & $\mathrm{Hm} 3$ & Oasato, Kikai, Kagoshima & 2006 \\
\hline & Kikai-269 & $\mathrm{Hm} 4$ & Oasato, Kikai, Kagoshima & 2007 \\
\hline & Kikai-301 & Hm5 & Oasato, Kikai, Kagoshima & 2007 \\
\hline & Kikai-318 & Hm6 & Oasato, Kikai, Kagoshima & 2007 \\
\hline & Kikai-323 & $\mathrm{Hm} 7$ & Oasato, Kikai, Kagoshima & 2007 \\
\hline \multirow[t]{16}{*}{ 2) Tokunoshima Island } & Toku-225 & $\mathrm{Hm} 8$ & Kinen, Isen, Tokunoshima, Kagoshima & 2006 \\
\hline & Toku-228 & $\mathrm{Hm} 9$ & Kinen, Isen, Tokunoshima, Kagoshima & 2006 \\
\hline & Toku-229 & Hm10 & Kinen, Isen, Tokunoshima, Kagoshima & 2006 \\
\hline & Toku-230 & Hm11 & Kinen, Isen, Tokunoshima, Kagoshima & 2006 \\
\hline & Toku-231 & $\mathrm{Hm} 12$ & Kinen, Isen, Tokunoshima, Kagoshima & 2006 \\
\hline & Toku-232 & $\mathrm{Hm} 13$ & Kinen, Isen, Tokunoshima, Kagoshima & 2006 \\
\hline & Toku-233 & $\mathrm{Hm} 14$ & Kinen, Isen, Tokunoshima, Kagoshima & 2006 \\
\hline & Toku-234 & $\operatorname{Hm} 15$ & Nishi-metegu, Isen, Tokunoshima, Kagoshima & 2006 \\
\hline & Toku-235 & $\mathrm{Hm} 16$ & Higashi-metegu, Isen, Tokunoshima, Kagoshima & 2006 \\
\hline & Toku-236 & Hm17 & Higashi-metegu, Isen, Tokunoshima, Kagoshima & 2006 \\
\hline & Toku-237 & $\operatorname{Hm} 18$ & Higashi-metegu, Isen, Tokunoshima, Kagoshima & 2006 \\
\hline & Toku-238 & Hm19 & Higashi-metegu, Isen, Tokunoshima, Kagoshima & 2006 \\
\hline & Toku-239 & $\operatorname{Hm} 20$ & Higashi-metegu, Isen, Tokunoshima, Kagoshima & 2006 \\
\hline & Toku-240 & $\operatorname{Hm} 21$ & Saben, Isen, Tokunoshima, Kagoshima & 2006 \\
\hline & Toku-241 & $\operatorname{Hm} 22$ & Saben, Isen, Tokunoshima, Kagoshima & 2006 \\
\hline & Toku-244 & $\operatorname{Hm} 23$ & Saben, Isen, Tokunoshima, Kagoshima & 2006 \\
\hline \multirow[t]{4}{*}{ 3) Yoron Island } & Yoron-57 & H1 & Yoron, Kagoshima & 2002 \\
\hline & Yoron-83 & $\mathrm{H} 2$ & Yoron, Kagoshima & 2002 \\
\hline & Yoron-121 & $\mathrm{H} 3$ & Yoron, Kagoshima & 2002 \\
\hline & Yoron-127 & $\mathrm{H} 4$ & Yoron, Kagoshima & 2002 \\
\hline \multirow[t]{9}{*}{ 4) Okinawa Main Island } & KIN-1 & Iw2 & Kin, Okinawa & 1994 \\
\hline & Honto-4 & Iw5 & Okinawa & 2005 \\
\hline & KIN-3 & Ns1 & Kin, Okinawa & 2007 \\
\hline & Ishi-2 & Ns2 & Ishikawa, Uruma, Okinawa & 2007 \\
\hline & Nago-Nc-1 & K14 & Nago, Okinawa & 2007 \\
\hline & Nago-4 & K15 & Nago, Okinawa & 2007 \\
\hline & MotobuB-1 & K16 & Motobu, Okinawa & 2007 \\
\hline & Kin2-1 & K17 & Kin, Okinawa & 2007 \\
\hline & Nakijin-5 & K18 & Nakijin, Okinawa & 2007 \\
\hline
\end{tabular}


Table 1. (continued)

\begin{tabular}{|c|c|c|c|c|}
\hline & Isolate & Code & Location & Year of collection \\
\hline & HigashiA-3 & K19 & Higashi, Okinawa & 2007 \\
\hline & OgimiA-3 & K20 & Ogimi, Okinawa & 2007 \\
\hline & Uruma1-1 & K21 & Gushikawa, Uruma, Okinawa & 2007 \\
\hline & UrumaKA-5 & K22 & Katuren, Uruma, Okinawa & 2007 \\
\hline & A-17 & K23 & Okinawa, Okinawa & 2007 \\
\hline & A2-12 & K24 & Okinawa, Okinawa & 2007 \\
\hline & B-8 & K25 & Okinawa, Okinawa & 2007 \\
\hline & A-11 & K26 & Tomigusuku, Okinawa & 2007 \\
\hline & $\mathrm{C}-3$ & K27 & Itoman, Okinawa & 2007 \\
\hline & A-3 & K28 & Naha, Okinawa & 2007 \\
\hline & Hae-5 & K29 & Haebaru, Okinawa & 2007 \\
\hline & KO-7 & K30 & Kochinda, Yaese, Okinawa & 2007 \\
\hline \multirow[t]{4}{*}{ 5) Miyako Island } & Miyako-13 & Iw4 & Miyakojima, Okinawa & 2007 \\
\hline & S-2-4 & K1 & Shimoji, Miyakojima, Okinawa & 2007 \\
\hline & $\mathrm{H}-3$ & K3 & Hirara, Miyakojima, Okinawa & 2007 \\
\hline & U-4 & K4 & Ueno, Miyakojima, Okinawa & 2007 \\
\hline 6) Irabu Island & $\mathrm{I}-1$ & $\mathrm{~K} 2$ & Irabu, Miyakojima, Okinawa & 2007 \\
\hline \multirow[t]{6}{*}{ 7) Ishigaki Island } & Ishi-1 & Iw3 & Ishigaki, Okinawa & 2005 \\
\hline & Ishigaki-16 & Iw6 & Ishigaki, Okinawa & 2007 \\
\hline & Hirakubo-5 & K5 & Hirakubo, Ishigaki, Okinawa & 2007 \\
\hline & Hirano-4 & K6 & Hirano, Ishigaki, Okinawa & 2007 \\
\hline & Kawahara-2 & K7 & Kawahara, Ishigaki, Okinawa & 2007 \\
\hline & Hirae-1 & K8 & Hirae, Ishigaki, Okinawa & 2007 \\
\hline 8) Hateruma Island & Hateruma-1 & K9 & Hateruma, Taketomi, Okinawa & 2007 \\
\hline 9) Kohama Island & Kohama-4 & K10 & Kohama, Taketomi, Okinawa & 2007 \\
\hline 10) Yonaguni Island & Higawa-1 & K11 & Higawa, Yonaguni, Okinawa & 2007 \\
\hline 11) Tarama Island & Tarama-12 & $\mathrm{K} 12$ & Tarama, Okinawa & 2007 \\
\hline 12) Iheya Island & Iheya-2 & K13 & Iheya, Okinawa & 2007 \\
\hline 13) Iriomote Island & OK-901 & Iw1 & Iriomote, Taketomi, Okinawa & 1988 \\
\hline
\end{tabular}

isolates without DNA pol produce only one DNA band of $n u s \mathrm{G}-r p l \mathrm{~K}$ operon. We named these two genotypes as genotype I and II, respectively. Both genotype I and II were observed among 65 isolates analyzed in this study (Figs. $1 \& 2$ ). For example, the result of Fig. 2E showed that the isolates of Hateruma-1, Kohama-4, Higawa-1, and Tarama-12 were classified as genotype I, while the isolate of Iheya-2 as genotype II. Nine isolates belonged to geno- type I, and these isolates were collected from Hateruma, Irabu, Kohama, Miyako, Tarama, and Yonaguni Islands, which are relatively close to Taiwan (Fig. 1, 2D \& 2E). The other 56 isolates belonged to genotype II, which were collected from Iheya, Iriomote, Ishigaki, Kikai, Okinawa Main, Tokunoshima, and Yoron Islands (Figs. $1 \& 2 \mathrm{~A}$ to $2 \mathrm{C})$. The relationship between the year of collection and genotype could not be observed. For example, in spite of 


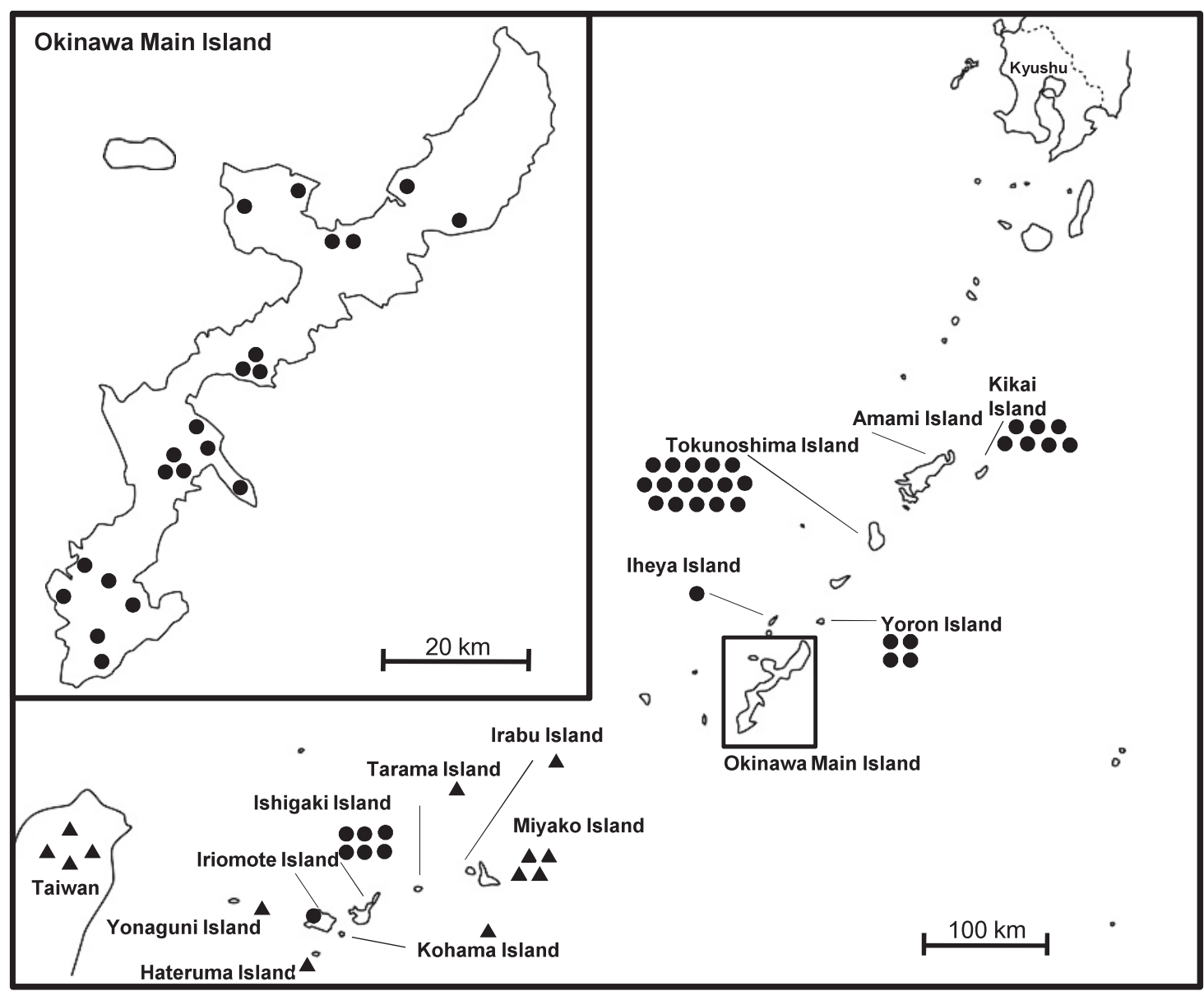

Fig. 1. Map of the Ryukyu Islands

An enlarged map of Okinawa Main Island is shown in the inset. The black triangles and circles show genotype I and II, respectively. Four Taiwanese isolates of genotype I are also shown.

the difference in year of collection, the isolates collected from Kikai and Okinawa Main Islands constituted the same genotype II.

The nine isolates of genotype I were sequenced around DNA pol. All the isolates sequenced in this study were 3,609 nucleotides long, and were identical among isolates. Moreover, sequence alignment was carried out using the sequences obtained in this study and the previously reported sequences of the Southeast Asian isolates ${ }^{16}$. Interestingly, the nine Japanese isolates sequenced in this study were identical with four Taiwanese isolates (TW2, TW3, TW5, and TW6, data not shown).

\section{Discussion}

Genetic diversity in bacteria can be assessed by examining specific restriction enzyme sites, repetitive elements, amplicons produced by random primers, or genome sequences ${ }^{11}$. Our previous study suggests that the polymorphic DNA pol may be useful for molecular differentiation among the Southeast Asian Las isolates ${ }^{16}$.
In this study, we used a duplex PCR of variable DNA pol and conserved $n u s \mathrm{G}-r p l \mathrm{~K}$ operon to differentiate among Las isolates of the Ryukyu Islands, and examined the genotypic distribution. Using this duplex PCR, the Las isolates collected from the Ryukyu Islands were clearly differentiated to two distinct genotypes. The genotype I in this study includes the group A, B and C that had been previously reported ${ }^{16}$, as well as the India-Poona isolate ${ }^{9}$. The genotype II consists of the Japanese isolates that lack DNA pol ${ }^{16}$. The method developed in this study could differentiate clearly among Las, especially genotype II.

Genotypic composition of Las isolates was different on each island. Especially, many Las isolates were collected from Okinawa Main and Tokunoshima Islands. These islands harbored only genotype II which is unique to Japan. These islands are the northern boundary of occurrence of greening from Southeast Asia. Dominance of unique isolates in the northern boundary region suggests that invasion of various foreign Las from southern Asian countries to these areas is not very frequent ${ }^{16}$. The Miyako-Yaemama area excluding Ishigaki and Iriomote 

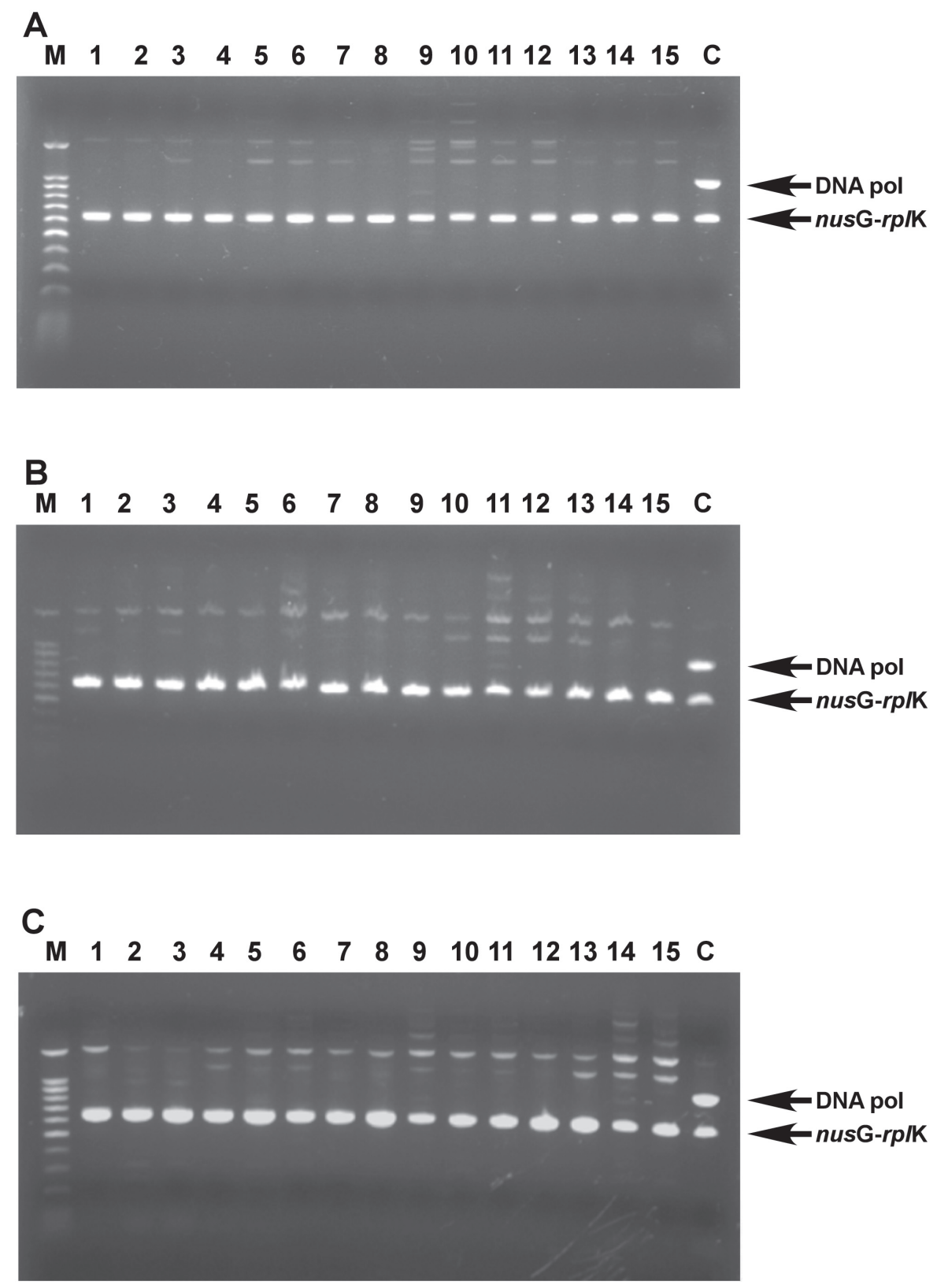

Fig. 2. Results of genotypes of Las isolates determined by the two primer pairs of the bacteriophage-type DNA polymerase region (DNA pol) and $n u s G-r p l K$ operon Arrows indicate that PCR amplicons of the DNA pol (988 bp, upper) and nusGrplK operon (627 bp, below). (A) Lane M, 100 bp Ladder (Promega). Lanes 1-7, Kikai-130, 145, 147, 269, 301, 318, and 323, respectively. Lanes 8-15, Toku-225, 228, 229, 230, 231, 232, 233 and 234, respectively. Lane C, TW2 (positive control). (B) Lane M, 100 bp Ladder. Lanes 1-8, Toku-235, 236, 237, 238, 239, 240, 241, and 244, respectively. Lanes 9-12, Yoron-57, 83, 121, and 127, respectively. Lanes 13-15, KIN-1, Honto-4, and KIN-3, respectively. Lane C, TW2. (C) Lane M, 100 bp Ladder. Lanes 1-15, Ishi-2, Nago-Nc-1, Nago-4, MotobuB-1, Kin2-1, Nakijin-5, HigashiA-3, OgimiA-3, Uruma1-1, UrumaKA-5, A-17, A2-12, B-8, A-11, and C-3, respectively. Lane C, TW2. (D) Lane M, 100 bp Ladder. Lanes 1-15, A-3, Hae-5, KO-7, Miyako-13, S-2-4, I-1, H-3, U-4, Ishi-1, Ishigaki-16, Hirakubo-5, Hirano-4, Kawahara-2, Hirae-1, and OK-901, respectively. Lane C, TW2. (E) Lane M, 100 bp Ladder. Lanes 1-5, Hateruma-1, Kohama-4, Higawa-1, Tarama-12, and Iheya-2, respectively. Lane C, TW2. 

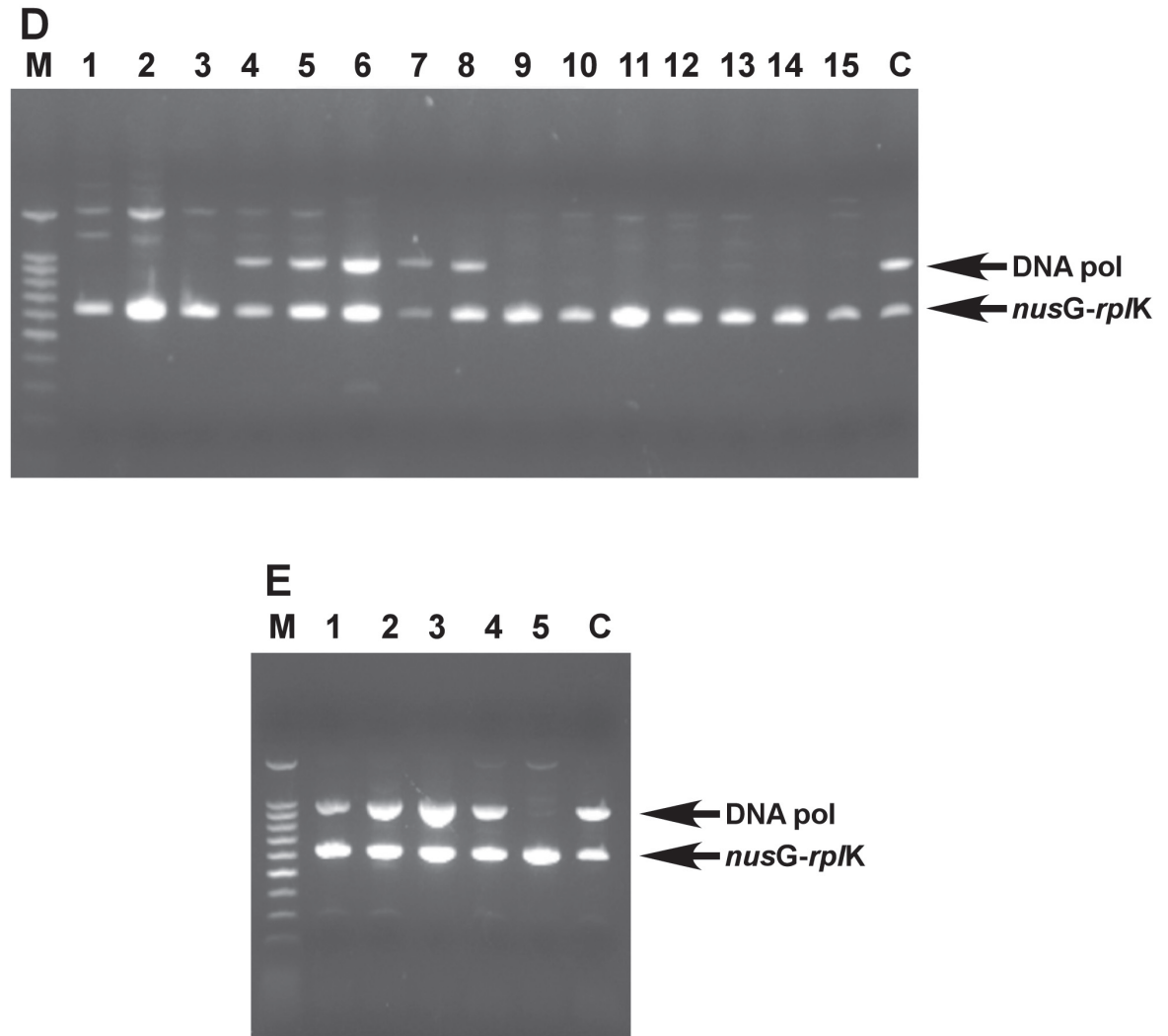

Fig. 2. (continued)

Islands in this survey apparently harbored only genotype I. However, the number of the isolates collected on these islands is insufficient to discuss the detailed within-island population structure. Another research survey would be needed to answer this question in the future.

All the genotype I isolates in the Ryukyu Islands sequenced in this study had identical DNA pol nucleotide sequences, moreover, these isolates were identical to four Taiwanese isolates. These results suggested that a part of the Las population in the Ryukyu Islands may share ancestral history with the Taiwanese population.

Citrus greening diseases are distributed mainly in tropical regions. Greening in Japan had been found first in the southernmost island of Iriomote, which is very close to Taiwan. The distribution of genotype I both in the Miyako-Yaeyama area and Taiwan suggests that some Las isolates may have been introduced from Taiwan to this region. On the other hand, the origin of genotype II, which is unique to Japan, remains unknown.

\section{Acknowledgments}

We thank Ms. H. Hatomi for meticulous technical assistance.

\section{References}

1. Aubert, B. (1987) Trioza erytreae Del Guercio and Diaphorina citri Kuwayama (Homoptera: Psylloidea), the two vectors of citrus greening disease: Biological aspects and possible control strategies. Fruits, 42, 149-162.

2. Bove, J. M. (2006) Huanglongbing: A destructive, newlyemerging, century-old disease of citrus. J. Plant Pathol., $\mathbf{8 8}, 7-37$.

3. Coletta, H. D. et al. (2005) Analysis of 16S rDNA sequences from citrus huanglongbing bacteria reveal a different "Ca. Liberibacter" strain associated with citrus disease in Sao Paulo. Plant Dis., 89, 848-852.

4. da Graça, J. V. (1991) Citrus greening disease. Annu. Rev. Phytopathol., 29, 109-136.

5. Halbert, S. E. \& Manjunath, K. L. (2004) Asian citrus psyllids (Sternorrhyncha: Psyllidae) and greening disease of citrus: a literature review and assessment of risk in Florida. Florida Entomol., 87, 330-353.

6. Hall, T. A. (1999) BIOEDIT: a user-friendly biological sequence alignment editor and analysis program for windows 95/98/NT. Nucleic Acids Symposium Series., 41, 95-98.

7. Hamashima, A. et al. (2003) First report of citrus greening disease in Kagoshima. Ann. Phytopathol. Soc. Jpn., 69, 307-308 [In Japanese].

8. Hoy, M. A., Jeyaprakash, A. \& Nguyen, R. (2001) Long PCR is a sensitive method for detecting Liberobacter asiaticum in parasitoids undergoing risk assessment in quarantine. Biol. Cont., 22, 278-287. 
9. Jagoueix, S., Bove, J. M. \& Garnier, M. (1997) Comparison of the $16 \mathrm{~S} / 23 \mathrm{~S}$ ribosomal intergenic regions of "Candidatus Liberobacter asiaticum" and "Candidatus Liberobacter africanum," the two species associated with citrus huanglongbing (greening) disease. Int. J. Syst. Bacteriol., 47, 224-227.

10. Kawano, S., Su, H. J. \& Uehara, K. (1997) First report of citrus greening disease in Okinawa island. Ann. Phytopathol. Soc. Jpn., 63, 256 [In Japanese].

11. Louws, F. J., Rademaker, J. L. W. \& de Bruijn, F. J. (1999) The three Ds of PCR-based genomic analysis of phytobacteria: diversity, detection, and disease diagnosis. Annu. Rev. Phytopathol., 37, 81-125.

12. Miyakawa, T. \& Tsuno, K. (1989) Occurrence of citrus greening disease in the southern islands of Japan. Ann. Phytopathol. Soc. Jpn., 55, 667-670.
13. Naito, T. et al. (2001) Detection of the citrus huanglongbing (greening disease) by polymerase chain reaction (PCR) assays and distribution in Okinawa, Japan. Bull. Okinawa Agric. Exp. Sta., 23, 74-81 [In Japanese with English summary].

14. Ooishi, T. et al. (2006) Geographical distribution of the Asian citrus psyllids infected with Candidatus Liberibacter asiaticus in Okinawa Prefecture. Kyushu Pl. Prot. Res., 52, 66-70 [In Japanese].

15. Shinohara, K. et al. (2006) Survey of citrus huanglongbing (greening disease) on the Amami Islands. 1. Characteristics of distribution in the Amami Islands. Kyushu Pl. Prot. Res., 52, 6-10 [In Japanese with English summary].

16. Tomimura, K. et al. (2009) Evaluation of genetic diversity among Candidatus Liberibacter asiaticus isolates collected in Southeast Asia. Phytopathology, 99, 1062-1069. 